\title{
A version of Hörmander's theorem for the fractional Brownian motion
}

\author{
Fabrice Baudoin \\ Laboratoire de Statistiques et Probabilités \\ Université Paul Sabatier \\ 118 Route de Narbonne, Toulouse, France \\ fbaudoin@cict.fr
}

\author{
Martin Hairer \\ Mathematics Institute \\ The University of Warwick \\ Coventry CV4 7AL, United Kingdom \\ hairer@maths.warwick.co.uk
}

July 31,2018

\begin{abstract}
It is shown that the law of an SDE driven by fractional Brownian motion with Hurst parameter greater than $1 / 2$ has a smooth density with respect to Lebesgue measure, provided that the driving vector fields satisfy Hörmander's condition. The main new ingredient of the proof is an extension of Norris' lemma to this situation.
\end{abstract}

\section{Contents}

1 Introduction 1

2 Malliavin calculus with respect to fractional Brownian motion 2

3 Norris' lemma for integrals with respect to fractional Brownian motion 5

4 Existence and smoothness of the density under Hörmander's condition 11

5 Asvmptotics of the density in small times 15

\section{Introduction}

In the celebrated paper [Hör67], L. Hörmander gave in 1967 a sufficient (and necessary in the analytic case) condition for the hypoellipticity of second order differential operators. The original proof of Hörmander was rather complicated and has been since considerably simplified in using the theory of pseudo-differential operators.

In 1976, P. Malliavin, uses the deep connection between the theory of second order differential operators and Itô's theory of stochastic differential equations to point out the probabilistic counterpart of Hörmander's theorem. The problem of the hypoellipticity is essentially equivalent to the 
problem of the existence of a smooth density with respect to the Lebesgue measure for the solution of a stochastic differential equation. The idea of Malliavin's proof of Hörmander's theorem is to show that the Itô's map associated with a stochastic differential equation is differentiable in a weak sense and then to show that, under Hörmander's conditions, this derivative is non-degenerate. The stochastic calculus of variations that has been developed in [Mal78] precisely in order to obtain a probabilistic proof of Hörmander's theorem is now known as Malliavin calculus and has since then found numerous applications (see [Nua95]).

In the last few years, there have been numerous attempts to define a notion of solution for differential equations driven by a fractional Brownian motion. When the Hurst parameter of the fractional Brownian motion is greater than $1 / 2$, existence and uniqueness of the solution are obtained by Zähle in [Zäh01] or Nualart-Răşcanu in [NR02]. Let us note that, as a consequence of the work of Coutin and Qian [CQ02], a notion of solution can actually be well-defined for $H>\frac{1}{4}$. The problem of the existence and smoothness of the density with respect to Lebesgue measure for solutions of stochastic differential equations that are driven by a fractional Brownian motion with Hurst parameter greater than $1 / 2$ is solved in some special cases. In [NT06] the existence and smoothness of the density has been shown in the one-dimensional case by using Doss-Süssman methods. In [NS05], the authors prove the existence of a density under ellipticity assumptions. Finally, in [HN06], always under an ellipticity assumption, the smoothness of the density is proved.

In the present work we prove a version of Hörmander's theorem for solutions of differential equations driven by a fractional Brownian motion. More precisely, we prove that as soon as the usual Hörmander conditions are satisfied, there exists a smooth density for the law of the solutions. One of the major difficulties is to prove an equivalent for fractional Brownian motion of Norris' lemma [Nor86]. This lemma, interesting in itself, quantifies in which way the integrand of a stochastic integral with respect to fractional Brownian motion has to be small if the stochastic integral is small.

This article is organised as follows. In a first section we recall the basics of Malliavin calculus in the context of fractional Brownian motion, which is the main tool used in this article. We then prove a version of Norris' lemma for stochastic integrals with respect to fractional Brownian motion. This lemma is then used to prove the main result of this work, that is the existence and the smoothness of the density under Hörmander's conditions. Finally, in a last section, we apply this result to the analysis of the behaviour in small times of this density on the diagonal.

\section{Acknowledgements}

The authors would like to thank L. Coutin and P. Friz for stimulating discussions.

\section{Malliavin calculus with respect to fractional Brownian motion}

Let us first recall some basic facts about Malliavin calculus with respect to the fractional Brownian motion (for further details, we refer for instance to [HN06] or [NS05]).

We consider the Wiener space of continuous paths:

$$
\mathbb{W}^{\otimes d}=\left(\mathcal{C}\left([0,1], \mathbb{R}^{d}\right),\left(\mathcal{B}_{t}\right)_{0 \leq t \leq 1}, \mathbb{P}\right)
$$


where:

1. $\mathcal{C}\left([0,1], \mathbb{R}^{d}\right)$ is the space of continuous functions $[0,1] \rightarrow \mathbb{R}^{d}$;

2. $\left(\beta_{t}\right)_{t>0}$ is the coordinate process defined by $\beta_{t}(f)=f(t), f \in \mathcal{C}\left([0,1], \mathbb{R}^{d}\right)$;

3. $\mathbb{P}$ is the Wiener measure;

4. $\left(\mathcal{B}_{t}\right)_{0 \leq t \leq 1}$ is the $\left(\mathbb{P}\right.$-completed) natural filtration of $\left(\beta_{t}\right)_{0 \leq t \leq 1}$.

A $d$-dimensional fractional Brownian motion with Hurst parameter $H \in(0,1)$ is a Gaussian process

$$
B_{t}=\left(B_{t}^{1}, \ldots, B_{t}^{d}\right), t \geq 0,
$$

where $B^{1}, \ldots, B^{d}$ are $d$ independent centred Gaussian processes with covariance function

$$
R(t, s)=\frac{1}{2}\left(s^{2 H}+t^{2 H}-|t-s|^{2 H}\right) .
$$

It can be shown that such a process admits a continuous version whose paths are Hölder $p$ continuous, $p<H$. The fractional Brownian motion with Hurst parameter $H>\frac{1}{2}$ can be constructed on the Wiener space by a Volterra type representation. Namely, the process

$$
B_{t}=\int_{0}^{t} \mathbf{K}(t, s) d \beta_{s}, t \geq 0
$$

is a fractional Brownian motion with Hurst parameter $H$, where

$$
\mathbf{K}(t, s)=c_{H} s^{\frac{1}{2}-H} \int_{s}^{t}(u-s)^{H-\frac{3}{2}} u^{H-\frac{1}{2}} d u, \quad t>s .
$$

and $c_{H}$ is a suitable constant. Let $\mathcal{E}$ be the space of $\mathbb{R}^{d}$-valued step functions on $[0,1]$. We denote by $\mathcal{H}$ the closure of $\mathcal{E}$ for the scalar product:

$$
\left\langle\left(\mathbf{1}_{\left[0, t_{1}\right]}, \cdots, \mathbf{1}_{\left[0, t_{d}\right]}\right),\left(\mathbf{1}_{\left[0, s_{1}\right]}, \cdots, \mathbf{1}_{\left[0, s_{d}\right]}\right)\right\rangle_{\mathcal{H}}=\sum_{i=1}^{d} R\left(t_{i}, s_{i}\right) .
$$

For $\varphi, \psi \in \mathcal{H}$, we have

$$
\langle\varphi, \psi\rangle_{\mathcal{H}}=H(2 H-1) \int_{0}^{1} \int_{0}^{1}|s-t|^{2 H-2}\langle\varphi(s), \psi(s)\rangle_{\mathbb{R}^{d}} d s d t .
$$

It can be shown that $L^{1 / H}\left([0,1], \mathbb{R}^{d}\right) \subset \mathcal{H}$ but that $\mathcal{H}$ also contains distributions (see for example [PT00]).

For our purposes, the following representation of the $\mathcal{H}$-scalar product is useful. Let $\mathcal{I}^{\alpha} \varphi$ denote the fractional integral of order $\alpha$ of $\varphi$, defined by

$$
\mathcal{I}^{\alpha} \varphi(t)=\frac{1}{\Gamma(\alpha)} \int_{0}^{t}(t-s)^{\alpha-1} \varphi(s) d s
$$


If one extends $\varphi$ and $\psi$ to $\mathbb{R}_{+}$by setting $\varphi(t)=\psi(t)=0$ for $t \geq 1$, a straightforward application of Fubini's theorem shows that one has the identity

$$
\langle\varphi, \psi\rangle_{\mathcal{H}}=\left\langle\mathcal{I}^{H-1 / 2} \varphi, \mathcal{I}^{H-1 / 2} \psi\right\rangle_{L^{2}} .
$$

A $\mathcal{B}_{1}$-measurable real valued random variable $F$ is said to be cylindrical if it can be written as

$$
F=f\left(\int_{0}^{1}\left\langle h_{s}^{1}, d B_{s}\right\rangle, \ldots, \int_{0}^{1}\left\langle h_{s}^{n}, d B_{s}\right\rangle\right),
$$

where $h^{i} \in \mathcal{H}$ and $f: \mathbb{R}^{n} \rightarrow \mathbb{R}$ is a $C^{\infty}$ bounded function. The set of cylindrical random variables is denoted $\mathcal{S}$. The Malliavin derivative of $F \in \mathcal{S}$ is the $\mathbb{R}^{d}$ valued stochastic process $\left(\mathbf{D}_{t} F\right)_{0 \leq t \leq 1}$ given by

$$
\mathbf{D}_{t} F=\sum_{i=1}^{n} h^{i}(t) \frac{\partial f}{\partial x_{i}}\left(\int_{0}^{1}\left\langle h_{s}^{1}, d B_{s}\right\rangle, \ldots, \int_{0}^{1}\left\langle h_{s}^{n}, d B_{s}\right\rangle\right) .
$$

More generally, we can introduce iterated derivatives. If $F \in \mathcal{S}$, we set

$$
\mathbf{D}_{t_{1}, \ldots, t_{k}}^{k} F=\mathbf{D}_{t_{1}} \ldots \mathbf{D}_{t_{k}} F \text {. }
$$

For any $p \geq 1$, the operator $\mathbf{D}^{k}$ is closable from $\mathcal{S}$ into $L^{p}\left(\mathcal{C}\left([0,1], \mathbb{R}^{d}\right), \mathcal{H}^{\otimes k}\right)$. We denote by $\mathbb{D}^{k, p}(\mathcal{H})$ the closure of the class of cylindrical random variables with respect to the norm

$$
\|F\|_{k, p}=\left(\mathbb{E}\left(F^{p}\right)+\sum_{j=1}^{k} \mathbb{E}\left(\left\|\mathbf{D}^{j} F\right\|_{\mathcal{H}^{\otimes j}}^{p}\right)\right)^{\frac{1}{p}},
$$

and

$$
\mathbb{D}^{\infty}(\mathcal{H})=\bigcap_{p \geq 1} \bigcap_{k \geq 1} \mathbb{D}^{k, p}(\mathcal{H})
$$

We also introduce the localised spaces $\mathbb{D}_{\text {loc }}^{k, p}(\mathcal{H})$ by saying that a random variable $F$ belongs to $\mathbb{D}_{\text {loc }}^{k, p}(\mathcal{H})$ if there exists a sequence of sets $\Omega_{n} \subset \mathcal{B}_{1}$ and random variables $F_{n} \in \mathbb{D}^{k, p}(\mathcal{H})$ such that $\Omega_{n} \uparrow \mathcal{C}\left([0,1], \mathbb{R}^{d}\right)$ almost surely and such that $F=F_{n}$ on $\Omega_{n}$.

We then have the following key result which stems from Theorem 2.1.2 and Corollary 2.1.2. in [Nua95:

Theorem 2.1 Let $F=\left(F_{1}, \ldots, F_{n}\right)$ be a $\mathcal{B}_{1}$-measurable random vector such that:

1. For every $i=1, \ldots, n, F_{i} \in \mathbb{D}_{\text {loc }}^{1,2}(\mathcal{H})$;

2. The matrix $\Gamma=\left(\left\langle\mathbf{D} F^{i}, \mathbf{D} F^{j}\right\rangle_{\mathcal{H}}\right)_{1 \leq i, j \leq n}$ is invertible almost surely.

Then the law of $F$ has a density with respect to the Lebesgue measure on $\mathbb{R}^{n}$. If moreover $F \in$ $\mathbb{D}^{\infty}(\mathcal{H})$ and, for every $p>1$,

$$
\mathbb{E}\left(\frac{1}{|\operatorname{det} \Gamma|^{p}}\right)<+\infty,
$$

then this density is smooth.

Remark 2.2 The matrix $\Gamma$ is called the Malliavin matrix of the random vector $F$. 


\section{Norris' lemma for integrals with respect to fractional Brownian motion}

A main ingredient in many probabilistic proofs of Hörmander's theorem (see for example [Nua95]) is Norris' lemma [Nor86]. Loosely speaking, it is a more quantitative version of the uniqueness property of the semimartingale decomposition, stating that if a semimartingale is small, then both its bounded variation part and its martingale part must be small. In other words, the martingale part and the bounded variation part cannot compensate each other. This section is devoted to the proof of Proposition 3.4 which is a version of Norris' lemma formulated in a framework suitable for the purposes of this article.

We start by stating a minor variant of a well-known concentration result for Gaussian measures.

Lemma 3.1 Let $\mu$ be a Gaussian measure on a separable Hilbert space $\mathcal{H}$ with covariance operator $\Gamma$ and write $T=\sqrt{\operatorname{tr} \Gamma}$ and $\lambda=\sqrt{\|\Gamma\|}$. Then, there exists a constant $C$ independent of $\Gamma$ such that one has the bound

$$
\mu(|\|x\|-T| \geq h) \leq C \exp \left(-\frac{h^{2}}{4 \lambda^{2}}\right),
$$

for every $h \geq 0$.

Proof. We can assume $\mathcal{H}$ to be finite-dimensional; the general case follows from a simple approximation argument since none of the constants depends on the dimension of $\mathcal{H}$. Define $\tilde{T}=$ $\int\|x\| \mu(d x)$. It follows from the isoperimetric inequality for Gaussian measures [CIS76, Tal95] that

$$
\mu(|\|x\|-\tilde{T}| \geq h) \leq C \exp \left(-\frac{h^{2}}{2 \lambda^{2}}\right),
$$

for every $h \in \mathbb{R}$. Furthermore, it follows from [Bog98 Thm 1.7.1] that

$$
T^{2}-\tilde{T}^{2} \leq \frac{\pi^{2}}{4} \lambda^{2}, \quad \text { and thus } \quad T-\tilde{T} \leq \frac{\pi^{2} \lambda^{2}}{4 T} \leq \frac{\pi^{2} \lambda}{4} .
$$

The claim follows at once.

We now define the class of Gaussian processes that are of interest to us. For $H \in\left(\frac{1}{2}, 1\right]$, we say that a Gaussian process $B$ is of type $H$ if it is centred and the function $f$ defined by

$$
f(s, t)=\mathbb{E}(B(t)-B(s))^{2}
$$

is $\mathcal{C}^{1}$ and satisfies

$$
c_{1}|t-s|^{2 H} \leq f(s, t) \leq c_{2}|t-s|^{2 H}, \quad\left|\partial_{s} \partial_{t} f(s, t)\right| \leq c_{3}|t-s|^{2 H-2},
$$

for every pair of times $s, t \in(0,1)$ with $s \neq t$. The following is a direct consequence of (3.5) combined with 3.1 
Lemma 3.2 Let $B^{i}$ be i.i.d. Gaussian processes of type $H>\frac{1}{2}$ and let $\delta, N>0$ be such that $\delta N<1$. Define the $\mathbb{R}^{N}$-valued random variables $X^{i}$ and the number $T$ by

$$
X_{n}^{i}=B^{i}(n \delta)-B^{i}((n-1) \delta), \quad T^{2}=\sum_{n=1}^{N} f(n \delta,(n-1) \delta) .
$$

Then, there exist constants $C_{1}, C_{2}$ such that one has the bound

$$
\begin{gathered}
\mathbb{P}\left(|| X^{i}|-T| \geq h\right) \leq C_{1} \exp \left(-C_{2} \frac{N h^{2}}{(\delta N)^{2 H}}\right), \\
\mathbb{P}\left(\left|\left\langle X^{i}, X^{j}\right\rangle\right| \geq h^{2}\right) \leq C_{1} \exp \left(-C_{2} \frac{N h^{2}}{(\delta N)^{2 H}}\right),
\end{gathered}
$$

for every $h \geq 0$ and every pair $(i, j)$.

Proof. Denote by $\Gamma$ the covariance of $X^{i}$ (this is independent of $i$ ). Then, one has

$$
\Gamma_{m n}=\frac{1}{2} \int_{m \delta}^{(m+1) \delta} \int_{n \delta}^{(n+1) \delta} \partial_{s} \partial_{t} f(s, t) d s d t,
$$

so that $\left|\Gamma_{m n}\right| \leq C \delta^{2 H}(1+|m-n|)^{2 H-2}$. This implies that

$$
\|\Gamma\|_{\mathrm{HS}}^{2}=\sum_{m, n=1}^{N}\left|\Gamma_{m n}\right|^{2} \leq C N \delta^{4 H} \sum_{k=1}^{N}|k|^{4 H-4} \leq C \delta^{4 H} N^{4 H-2} .
$$

Since this is a bound on $\|\Gamma\|^{2}$, the first inequality follows from Lemma 3.1 Fix now an arbitrary pair of indices $i \neq j$. Note that conditional on the value of $X^{j}$, the random variable $\left\langle X^{i}, X^{j}\right\rangle$ is normal with variance $\left\langle X^{j}, \Gamma X^{j}\right\rangle$. This motivates the introduction of the random vector $\bar{X}^{j}=$ $\Gamma^{1 / 2} X^{j}$ which is Gaussian with covariance $\Gamma^{2}$. Note also that $\mathbb{E}\left\|\bar{X}^{j}\right\|^{2}=\operatorname{tr}\left(\Gamma^{2}\right)=\|\Gamma\|_{\mathrm{HS}}^{2}$. We thus have, for any $v>0$, the bound

$$
\begin{aligned}
\mathbb{P}\left(\left|\left\langle X^{i}, X^{j}\right\rangle\right| \geq h^{2}\right) & =\mathbb{E}\left(\mathbb{P}\left(\left|\left\langle X^{i}, X^{j}\right\rangle\right| \geq h^{2} \mid X^{j}\right)\right) \leq \mathbb{E}\left(C \exp \left(-h^{4} /\left\|\bar{X}^{j}\right\|^{2}\right)\right) \\
& \leq C \exp \left(-\frac{h^{4}}{4\left(\|\Gamma\|_{\mathrm{HS}}+v\right)^{2}}\right)+\mathbb{P}\left(\left|\left\|\bar{X}^{j}\right\|-\|\Gamma\|_{\mathrm{HS}}\right| \geq v\right) \\
& \leq C \exp \left(-\frac{h^{4}}{8\left(\|\Gamma\|_{\mathrm{HS}}^{2}+v^{2}\right)}\right)+C \exp \left(-\frac{v^{2}}{4\left\|\Gamma^{2}\right\|}\right) \\
& \leq C \exp \left(-\frac{h^{4}}{8\left(\|\Gamma\|_{\mathrm{HS}}^{2}+v^{2}\right)}\right)+C \exp \left(-\frac{v^{2}}{4\|\Gamma\|_{\mathrm{HS}}^{2}}\right) .
\end{aligned}
$$

Note now that the second inequality is non-trivial only for $h^{2} \geq\|\Gamma\|_{\mathrm{HS}}$, so that we assume that we are in this situation from now on. Choosing $v^{2}=h^{2}\|\Gamma\|_{\text {HS }}$, we get

$$
\mathbb{P}\left(\left|\left\langle X^{i}, X^{j}\right\rangle\right| \geq h^{2}\right) \leq C \exp \left(-h^{2} /\left(16\|\Gamma\|_{\mathrm{HS}}\right)\right)
$$

which, together with 3.8 , implies the required bound. 
Remark 3.3 The bounds in Lemma 3.2 can be interpreted as saying that the 'coarse-grained quadratic variation' of $B$ on a scale $\delta$ and over a time interval $t$ behaves like $\delta^{2 H-1} t$ to within an error of order $\delta^{H} t^{H}$. Note that the relative magnitude of the error to the average value always tends to 0 as $\delta \rightarrow 0$, but that this ratio becomes 'worse' as $H \rightarrow 1$.

We now have the main tools in place to prove the following version of Norris' lemma. Note that here and in the sequel, we denote by $\|\cdot\|_{\alpha}$ the $\alpha$-Hölder norm of a function.

Proposition 3.4 Let $H \in\left(\frac{1}{2}, 1\right)$ and let $a$ and $b$ be processes taking values in $\mathbb{R}$ and $\mathbb{R}^{m}$ respectively such that $\mathbb{E}\left(\|a\|_{\tilde{H}}+\sum_{i}\left\|b^{i}\right\|_{\tilde{H}}\right)^{p}<\infty$ for every $p \geq 1$ and every $\tilde{H} \in\left(\frac{1}{2}, H\right)$. Let

$$
y_{t}=\int_{0}^{t} a(s) d s+\int_{0}^{t}\langle b(s), d B(s)\rangle,
$$

where the $B_{i}$ are $m$ i.i.d. Gaussian process of type $H$. Then there exists $q>0$ such that, for every $p>0$, the estimate

$$
\mathbb{P}\left(\|y\|_{L^{\infty}}<\epsilon \quad \text { and } \quad\|a\|_{L^{\infty}}+\|b\|_{L^{\infty}}>\epsilon^{q}\right)<C_{p} \epsilon^{p}
$$

holds. The constant $C_{p}$ depends on $a, b$, and $p$ but not on $\epsilon$.

Remark 3.5 Note that we do not require the $B_{i}$ to be independent of the processes $a$ and $b$. We also do not require any adaptedness at this stage. The reason why we will require adaptedness later on is that equation (4.21) for the Malliavin derivative of the solution does not hold otherwise.

Remark 3.6 The bound (3.10) actually implies the bound

$$
\mathbb{P}\left(\|y\|_{L^{\infty}}<\epsilon\right) \leq C_{p} \varepsilon^{p}+\min \left\{\mathbb{P}\left(\|a\|_{L^{\infty}}<\varepsilon^{q}\right), \mathbb{P}\left(\|b\|_{L^{\infty}}<\varepsilon^{q}\right)\right\},
$$

which will be used repeatedly in the sequel.

Proof of Proposition 3.4 The proof consists of two parts. In the first part, we show that one has a bound of the type

$$
\mathbb{P}\left(\|y\|_{L^{\infty}}<\epsilon \quad \text { and } \quad\|b\|_{L^{\infty}}>\epsilon^{q}\right)<C_{p} \epsilon^{p} .
$$

In the second part, we use this information to show that one has also

$$
\mathbb{P}\left(\|y\|_{L^{\infty}}<\epsilon \quad \text { and } \quad\|a\|_{L^{\infty}}>\epsilon^{q}\right)<C_{p} \epsilon^{p} .
$$

Combining both bounds then yields 3.10 .

The key idea to the proof of (3.12), which should be translated to if $y$ is small then $b$ must also be small' is the following. Choose two small length scales $\delta \ll \Delta \ll 1$. Since we assume some regularity on $b$, it is easy to control the error made by assuming that $b$ is constant on intervals of length $\Delta$. One then considers the square root of the coarse-grained quadratic variation of $y$ on a scale $\delta$ over an interval of size $\Delta$ around $t$. This is of course bounded by $\|y\|_{L^{\infty}} \delta^{-1 / 2} \Delta^{1 / 2}$. 
By Remark 3.5 the contribution of the term including $b$ to this expression is approximately equal to $\delta^{H-1 / 2} \Delta^{1 / 2}|b(t)|$. The contribution of the term including $a$ on the other hand is bounded by $\|a\|_{L^{\infty}} \delta^{1 / 2} \Delta^{1 / 2}$. Summing over all intervals of size $\Delta$ yields a bound of the type

$$
\|b\|_{L^{1}} \lesssim \delta^{-H}\|y\|_{L^{\infty}}+\delta^{1-H}\|a\|_{L^{\infty}},
$$

from which it is then straightforward to deduce (3.12) by making use of the a priori bounds on the Hölder norms of $a$ and $b$.

This argument is of course extremely sloppy, since we have not justified in any way some of the approximations made and we have not addressed the fact that $b$ takes values in $\mathbb{R}^{m}$. Fix some small value of $\epsilon>0$ and fix two small numbers $\delta$ and $\Delta$ such that $1 / \delta$ and $1 / \Delta$ are integers, $1 / \Delta$ divides $1 / \delta$, and such that $\delta \ll \Delta \ll 1$. We also fix $\tilde{H} \in\left(\frac{1}{2}, H\right)$ to be determined later. We define $\bar{b}(t)$ as the stepfunction with steps of length $\Delta$ approximating $b(t), \bar{b}(t)=b(\Delta[t / \Delta])$ and we write $\beta(t)=b(t)-\bar{b}(t)$. The stochastic integral of $\beta$ is bounded as follows.

Lemma 3.7 There exists a constant $C$ such that, for every $t \geq 0$ and every $s \in[0, \Delta]$, one has

$$
\left|\int_{t}^{t+s}\langle\beta(r), d B(r)\rangle\right| \leq C\|b\|_{\tilde{H}}\|B\|_{\tilde{H}} \Delta^{\tilde{H}} s^{\tilde{H}} .
$$

Proof. Since $s \leq \Delta$, we can assume without loss of generality that $\bar{b}$ is constant on the interval $[t, t+s]$. It follows from [You36] that, for every $\alpha>1$, there exists a constant $C$ such that

$$
\left|\int_{t}^{t+s}\langle\beta(r), d B(r)\rangle-\langle\beta(t), B(t+s)-B(t)\rangle\right| \leq C\|b\|_{\tilde{H}}\|B\|_{\tilde{H}} s^{2 \tilde{H}} .
$$

Furthermore, one has $|\langle\beta(t), B(t+s)-B(t)\rangle| \leq\|b\|_{\tilde{H}}\|B\|_{\tilde{H}} \Delta^{\tilde{H}} s^{\tilde{H}}$, so that the result follows at once.

Denote by $r$ the (integer) ratio $\Delta / \delta$, set $t_{n}=\delta n$ and define for $N=1, \ldots, \Delta^{-1}$ and for $i, j=1, \ldots, m$ the random variable

$$
X_{N}^{i j}=\sum_{n=(N-1) r}^{N r-1}\left(B_{i}\left(t_{n+1}\right)-B_{i}\left(t_{n}\right)\right)\left(B_{j}\left(t_{n+1}\right)-B_{j}\left(t_{n}\right)\right) .
$$

Note that $T^{2}:=\mathbb{E} X_{N}^{i i}=r f(\delta) \approx \Delta \delta^{2 H-1}$. With these notations and using Lemma 3.7 we have, for $n \in[(N-1) r, N r-1]$, the relation

$$
\begin{aligned}
\left|\left\langle b(N \Delta), B\left(t_{n+1}\right)-B\left(t_{n}\right)\right\rangle\right| & \leq\left|y_{t_{n+1}}-y_{t_{n}}\right|+\|a\|_{L^{\infty} \delta}+\left|\int_{t_{n}}^{t_{n+1}}\langle\beta(s), d B(s)\rangle\right| \\
& \leq 2\|y\|_{L^{\infty}}+\|a\|_{L^{\infty}} \delta+C\|b\|_{\tilde{H}}\|B\|_{\tilde{H}} \Delta^{\tilde{H}} \delta^{\tilde{H}} .
\end{aligned}
$$

Taking squares on both sides, summing from $n=(N-1) r$ to $n=N r-1$ and taking square roots, we get

$$
\sqrt{\sum_{i, j} b_{i}(N \Delta) b_{j}(N \Delta) X_{N}^{i j}} \leq \Delta^{1 / 2} \delta^{-1 / 2}\left(2\|y\|_{L^{\infty}}+\|a\|_{L^{\infty}} \delta+C\|b\|_{\tilde{H}}\|B\|_{\tilde{H}} \Delta^{\tilde{H}} \delta^{\tilde{H}}\right) .
$$


At this point, it is convenient to introduce quantities $Y_{N}^{i}=\left(X_{N}^{i i}\right)^{1 / 2}$ and $Y_{N}^{i j}=\left|X_{N}^{i j}\right|^{1 / 2}$. With this notation, we get

$$
\begin{aligned}
\sum_{i=1}^{m}\left|b_{i}(N \Delta)\right| Y_{N}^{i} \leq & C \sum_{i \neq j} Y_{N}^{i j} \sqrt{\left|b_{i}(N \Delta) b_{j}(N \Delta)\right|}+C \Delta^{1 / 2} \delta^{-1 / 2}\|y\|_{L^{\infty}} \\
& +C \Delta^{1 / 2} \delta^{1 / 2}\|a\|_{L^{\infty}}+C \Delta^{\tilde{H}+1 / 2} \delta^{\tilde{H}-1 / 2}\|b\|_{\tilde{H}}\|B\|_{\tilde{H}} .
\end{aligned}
$$

Summing over $N$ yields

$$
\begin{aligned}
\sum_{i=1}^{m} \sum_{N=1}^{\Delta^{-1}}\left|b_{i}(N \Delta)\right| Y_{N}^{i} \leq & C \sum_{i \neq j} \sum_{N=1}^{\Delta^{-1}} Y_{N}^{i j} \sqrt{\left|b_{i}(N \Delta) b_{j}(N \Delta)\right|}+C \Delta^{-1 / 2} \delta^{-1 / 2}\|y\|_{L^{\infty}} \\
& +C \Delta^{-1 / 2} \delta^{1 / 2}\|a\|_{L^{\infty}}+C \Delta^{\tilde{H}-1 / 2} \delta^{\tilde{H}-1 / 2}\|b\|_{\tilde{H}}\|B\|_{\tilde{H}} .
\end{aligned}
$$

Note now that by Lemma 3.1 one can hope that $Y_{N}^{i} \approx T$ for every $i$ and $N$. Therefore, the left hand side is very close to $T / \Delta$ times the sum of the $L^{1}$ norms of the $b_{i}$. More precisely, one has for every $i$, the bound

$$
\left|\Delta \sum_{N=1}^{\Delta^{-1}}\right| b_{i}(N \Delta)\left|-\left\|b_{i}\right\|_{L^{1}}\right| \leq\left\|b_{i}\right\|_{\tilde{H}} \Delta^{\tilde{H}},
$$

so that, multiplying 3.15 by $\Delta / T$, we get the bound

$$
\begin{aligned}
\sum_{i=1}^{m}\left\|b_{i}\right\|_{L^{1}} \leq & C \Delta^{\tilde{H}}\|b\|_{\tilde{H}}+C \delta^{-H}\|y\|_{L^{\infty}}+C \Delta^{\tilde{H}} \delta^{\tilde{H}-H}\|b\|_{\tilde{H}}\|B\|_{\tilde{H}} \\
& +C \delta^{1-H}\|a\|_{L^{\infty}}+C \Delta^{1 / 2} \delta^{1 / 2-H} \sum_{i=1}^{m} \sum_{N=1}^{\Delta^{-1}}\left|b_{i}(N \Delta) \| Y_{N}^{i}-T\right| \\
& +C \Delta^{1 / 2} \delta^{1 / 2-H} \sum_{i \neq j} \sum_{N=1}^{\Delta^{-1}} Y_{N}^{i j} \sqrt{\left|b_{i}(N \Delta) b_{j}(N \Delta)\right|} \\
\leq & C \Delta^{\tilde{H}}\|b\|_{\tilde{H}}+C \delta^{-H}\|y\|_{L^{\infty}}+C \Delta^{\tilde{H}} \delta^{\tilde{H}-H}\|b\|_{\tilde{H}}\|B\|_{\tilde{H}} \\
& +C \delta^{1-H}\|a\|_{L^{\infty}}+C \Delta^{1 / 2} \delta^{1 / 2-H}\|b\|_{\tilde{H}} \sum_{i, j=1}^{m} \sum_{N=1}^{\Delta^{-1}}\left|Y_{N}^{i j}-T \delta_{i j}\right|
\end{aligned}
$$

where we used $\delta_{i j}$ to denote the Kronecker delta. At this point, we note that, for every $\gamma \leq 1$, one has the interpolation inequality

$$
\|b\|_{L^{\infty}} \leq C\left(\gamma\|b\|_{\tilde{H}}+\gamma^{-1 / \tilde{H}}\|b\|_{L^{1}}\right) .
$$


Therefore

$$
\begin{aligned}
\|b\|_{L^{\infty}} \leq & C \gamma^{-1 / \tilde{H}} \Delta^{\tilde{H}}\|b\|_{\tilde{H}}+C \gamma^{-1 / \tilde{H}} \delta^{-H}\|y\|_{L^{\infty}}+C \gamma^{-1 / \tilde{H}} \delta^{1-H}\|a\|_{L^{\infty}} \\
& +C \gamma^{-1 / \tilde{H}} \Delta^{\tilde{H}} \delta^{\tilde{H}-H}\|b\|_{\tilde{H}}\|B\|_{\tilde{H}}+C \gamma\|b\|_{\tilde{H}} \\
& +C \gamma^{-1 / \tilde{H}} \Delta^{1 / 2} \delta^{1 / 2-H}\|b\|_{\tilde{H}} \sum_{i, j=1}^{m} \sum_{N=1}^{\Delta^{-1}}\left|Y_{N}^{i j}-T \delta_{i j}\right| .
\end{aligned}
$$

We now make the following choices for $\gamma, \delta$, and $\Delta$ :

$$
\gamma \approx \epsilon^{\frac{H(1-H)}{(1+H)(2-H)}}, \quad \delta \approx \epsilon^{\frac{1}{H(2-H)}}, \quad \Delta \approx \epsilon^{\frac{1-H}{H(2-H)}} .
$$

Note that, provided $\epsilon$ is small, one has indeed $\delta \ll \Delta \ll 1$. With these choices, and by choosing $\tilde{H}$ sufficiently close to $H$, we see that there exists a constant $\alpha>0$ and a constant $C>0$ such that

$$
\begin{aligned}
\|b\|_{L^{\infty}} & \leq C \epsilon^{\alpha}\left(\|b\|_{\tilde{H}}\left(1+\|B\|_{\tilde{H}}+\sum_{i, j, N} \frac{\left|Y_{N}^{i j}-T \delta_{i j}\right|}{\delta^{1 / 2} \Delta^{H-3 / 2}}\right)+\epsilon^{-1}\|y\|_{L^{\infty}}+\|a\|_{L^{\infty}}\right) \\
& \leq C \epsilon^{\alpha}\left(1+\|b\|_{\tilde{H}}^{2}+\|B\|_{\tilde{H}}^{2}+\left(\sum_{i, j, N} \frac{\left|Y_{N}^{i j}-T \delta_{i j}\right|}{\delta^{1 / 2} \Delta^{H-3 / 2}}\right)^{2}+\frac{\|y\|_{L^{\infty}}}{\epsilon}+\|a\|_{L^{\infty}}\right) .
\end{aligned}
$$

Actually, the constant $\alpha$ can be brought arbitrarily close to $\frac{H(1-H)}{(1+H)(2-H)} \geq \frac{2}{9}(1-H)$. Note that Lemma 3.2 yields the bound

$$
\mathbb{P}\left(\left|Y_{N}^{i j}-T \delta_{i j}\right|>h\right) \leq C \exp \left(-c \frac{h^{2}}{\delta \Delta^{2 H-1}}\right),
$$

for every possible value of $i, j$, and $N$. This immediately implies

$$
\mathbb{P}\left(\sum_{i, j, N} \frac{\left|Y_{N}^{i j}-T \delta_{i j}\right|}{\delta^{1 / 2} \Delta^{H-3 / 2}}>h\right) \leq \frac{C}{\Delta} \exp \left(-c h^{2}\right) .
$$

Therefore, there exists a constant $c$ such that, for $\epsilon$ small enough, one has the bound

$$
\begin{aligned}
\mathbb{P}\left(\|b\|_{L^{\infty}}>\epsilon^{\alpha / 2} \text { and }\|y\|_{L^{\infty}}<\epsilon\right) & \leq \mathbb{P}\left(\sum_{i, j, N} \frac{\left|Y_{N}^{i j}-T \delta_{i j}\right|}{\delta^{1 / 2} \Delta^{H-3 / 2}}>c \epsilon^{-\alpha / 4}\right) \\
& +\mathbb{P}\left(\|b\|_{\tilde{H}}^{2}+\|B\|_{\tilde{H}}^{2}+\|a\|_{L^{\infty}}>c \epsilon^{-\alpha / 2}\right) \leq C_{p} \epsilon^{p}
\end{aligned}
$$

The last inequality is obtained by combining 3.18 with the a priori bounds on the processes $a$, $b$, and $B$. We now turn to the proof of 3.13 . Fix again some small value of $\Delta$ to be determined 
later. We then have for every $t \in[0,1-\Delta]$ the inequality

$$
\begin{aligned}
\left|\int_{t}^{t+\Delta} a(s) d s\right| & \leq 2\|y\|_{L^{\infty}}+|\langle b(t), B(t+\Delta)-B(t)\rangle|+C\|b\|_{\tilde{H}}\|B\|_{\tilde{H}} \Delta^{2 \tilde{H}} \\
& \leq 2\|y\|_{L^{\infty}}+\|b\|_{L^{\infty}}\|B\|_{\tilde{H}} \Delta^{\tilde{H}}+C\|b\|_{\tilde{H}}\|B\|_{\tilde{H}} \Delta^{2 \tilde{H}} .
\end{aligned}
$$

It is easy to show that, similarly to 3.17 one has the inequality

$$
\|a\|_{L^{\infty}} \leq 2 \Delta^{-1} \sup _{t \in[0,1-\Delta]}\left|\int_{t}^{t+\Delta} a(s) d s\right|+2 \Delta^{\tilde{H}}\|a\|_{\tilde{H}},
$$

so that

$$
\begin{aligned}
\|a\|_{L^{\infty}} & \leq 2 \Delta^{-1}\|y\|_{L^{\infty}}+C\|b\|_{L^{\infty}}\|B\|_{\tilde{H}} \Delta^{\tilde{H}-1}+C\|b\|_{\tilde{H}}\|B\|_{\tilde{H}} \Delta^{2 \tilde{H}-1}+2 \Delta^{\tilde{H}}\|a\|_{\tilde{H}} \\
& \leq C\left(\Delta^{-1}\left(\|y\|_{L^{\infty}}+\|b\|_{L^{\infty}}^{2}\right)+\Delta^{2 \tilde{H}-1}\left(\|B\|_{\tilde{H}}^{2}+\|b\|_{\tilde{H}}^{2}+\|a\|_{\tilde{H}}\right)\right) .
\end{aligned}
$$

At this point, we choose $\Delta \approx \epsilon^{\alpha / H}$ with $\alpha$ as in 3.19 . This implies (by choosing as before $\tilde{H}$ sufficiently close to $H$ ) that there exists $\beta>0$ such that

$$
\|a\|_{L^{\infty}} \leq C \epsilon^{\beta}\left(\frac{\|y\|_{L^{\infty}}}{\epsilon}+\frac{\|b\|_{L^{\infty}}^{2}}{\epsilon^{\alpha}}+\|B\|_{\tilde{H}}^{2}+\|b\|_{\tilde{H}}^{2}+\|a\|_{\tilde{H}}\right) .
$$

Therefore, for $\epsilon$ small enough, there exists a constant $c$ such that

$$
\begin{aligned}
\mathbb{P}\left(\|a\|_{L^{\infty}}>\epsilon^{\beta / 2} \text { and }\|y\|_{L^{\infty}}<\epsilon\right) & \leq \mathbb{P}\left(\|b\|_{L^{\infty}}>\epsilon^{\alpha / 2} \text { and }\|y\|_{L^{\infty}}<\epsilon\right) \\
& +\mathbb{P}\left(\|B\|_{\tilde{H}}^{2}+\|b\|_{\tilde{H}}^{2}+\|a\|_{\tilde{H}}>c \varepsilon^{-\beta / 2}\right) \leq C_{p} \epsilon^{p},
\end{aligned}
$$

for arbitrary values of $p$. The last inequality is obtained by combining 3.19) with the a priori bounds on the processes $a, b$, and $B$. This concludes the proof of (3.13) and thus of Proposition 3.4

\section{Existence and smoothness of the density under Hörmander's con- dition}

We now arrive to the heart of our study and are interested in the study of the existence and regularity for the density of solutions of stochastic differential equations on $\mathbb{R}^{n}$

$$
X_{t}^{x}=x+\int_{0}^{t} V_{0}\left(X_{s}^{x}\right) d s+\sum_{i=1}^{d} \int_{0}^{t} V_{i}\left(X_{s}^{x}\right) d B_{s}^{i}
$$

where the $V_{i}$ 's are $C^{\infty}$-bounded vector fields on $\mathbb{R}^{n}$ and $B$ is the $d$ dimensional fractional Brownian motion defined by 2.1. For this type of equations, existence and uniqueness of the solution have been investigated by many authors (for instance Nualart-Rascânu in [NR02]). It has also been shown very recently in [HN06] that the law of the solution to 4.20] possesses a smooth density with respect to the Lebesgue measure in the elliptic case. Recall first the following a priori bound on the solutions to 4.20]. (See for example [NR02] for a proof.) 
Lemma 4.1 For every $\gamma<H$ and every $p>0, \mathbb{E}\left(\|X\|_{\gamma}^{p}\right)<+\infty$.

Let us now denote by $\Phi$ the stochastic flow associated with equation 4.20, that is $\Phi_{t}(x)=X_{t}^{x}$. From [HN06], we can deduce:

Lemma 4.2 The map $\Phi_{t}$ is $C^{1}$ and the first variation process defined by

$$
\mathbf{J}_{0 \rightarrow t}=\frac{\partial \Phi_{t}}{\partial x}
$$

satisfies the following equation:

$$
\mathbf{J}_{0 \rightarrow t}=\mathbf{I} \mathbf{d}_{\mathbb{R}^{n}}+\int_{0}^{t} D V_{0}\left(X_{s}^{x}\right) \mathbf{J}_{0 \rightarrow s} d s+\sum_{i=1}^{d} \int_{0}^{t} D V_{i}\left(X_{s}^{x}\right) \mathbf{J}_{0 \rightarrow s} d B_{s}^{i} .
$$

and, for every $p>1$,

$$
\mathbb{E}\left(\left\|\mathbf{J}_{0 \rightarrow 1}^{-1}\right\|^{p}\right)<+\infty .
$$

Furthermore, for every $i=1, \ldots, n, t>0$, and $x \in \mathbb{R}^{n}, X_{t}^{x, i} \in \mathbb{D}^{\infty}(\mathcal{H})$. Moreover,

$$
\mathbf{D}_{s}^{j} X_{t}^{x}=\mathbf{J}_{0 \rightarrow t} \mathbf{J}_{0 \rightarrow s}^{-1} V_{j}\left(X_{s}\right), \quad j=1, \ldots, d, \quad 0 \leq s \leq t,
$$

where $\mathbf{D}_{s}^{j} X_{t}^{x, i}$ is the $j$-th component of $\mathbf{D}_{s} X_{t}^{x, i}$.

We can now turn to our version of Hörmander's theorem for stochastic differential equations that are driven by a fractional Brownian motion with Hurst parameter $H>1 / 2$. If $I=$ $\left(i_{1}, \ldots, i_{k}\right) \in\{0, \ldots, d\}^{k}$, we denote by $V_{I}$ the Lie commutator defined by

$$
V_{I}=\left[V_{i_{1}},\left[V_{i_{2}}, \ldots,\left[V_{i_{k-1}}, V_{i_{k}}\right] \ldots\right] .\right.
$$

We also define the sets of vector fields

$$
\mathcal{V}_{n}=\left\{V_{I}, I \in\{0, \ldots, d\} \times\{1, \ldots, d\}^{n-1}\right\}, \quad \overline{\mathcal{V}}_{n}=\bigcup_{k=0}^{n} \mathcal{V}_{k}
$$

With these notations, the main result of this article is the following:

Theorem 4.3 Assume that, at some $x_{0} \in \mathbb{R}^{n}$, there exists $N$ such that

$$
\operatorname{span}\left\{V\left(x_{0}\right), V \in \overline{\mathcal{V}}_{N}\right\}=\mathbb{R}^{n} .
$$

Then, for any $t>0$, the law of the random variable $X_{t}^{x_{0}}$ has a smooth density with respect to the Lebesgue measure on $\mathbb{R}^{n}$.

Given the results from the previous sections, the proof of this theorem is by now quite standard and follows closely the argument given for instance in [Bis81], [Nor86] or [Nua95]. The main difference is that it is not a priori obvious how to relate the $L^{\infty}$ bounds obtained in Proposition 3.4 to the fractional Sobolev norms appearing in the statement of Theorem 2.1 
Lemma 4.4 Let $H>1 / 2$ and let $\mathcal{H}$ be defined as above. Then, for every $\gamma>H-1 / 2$ there exists a constant $C$ such that

$$
\|f\|_{\mathcal{H}} \geq C \frac{\|f\|_{L^{\infty}}^{3+1 / \gamma}}{\|f\|_{\gamma}^{2+1 / \gamma}},
$$

for every continuous function $f \in \mathcal{H}$. Here, $\|f\|_{\gamma}$ denotes as before the $\gamma$-Hölder norm of $f$.

Proof. Let $\mathcal{D}^{\alpha} f$ denote the fractional derivative of order $\alpha$ of $f$, defined by

$$
\mathcal{D}^{\alpha} f(t)=\frac{1}{\Gamma(1-\alpha)} \frac{d}{d t} \int_{0}^{t}(t-s)^{-\alpha} f(s) d s .
$$

We also introduce the operator $\mathcal{D}_{-}^{\alpha}$ defined by

$$
\mathcal{D}_{-}^{\alpha} f(t)=-\frac{\alpha}{\Gamma(1-\alpha)} \int_{t}^{\infty}(s-t)^{-\alpha-1}(f(s)-f(t)) d s,
$$

which is nothing but the adjoint of $\mathcal{D}^{\alpha}$ in $L^{2}\left(\mathbb{R}_{+}\right)$.

Since $\mathcal{I}^{\alpha}$ and $\mathcal{D}^{\alpha}$ are each other's inverse [SKM93], 2.3) implies by Cauchy-Schwartz that

$$
\left|\langle f, g\rangle_{L^{2}}\right|=\left|\left\langle\mathcal{I}^{H-1 / 2} f, \mathcal{D}_{-}^{H-1 / 2} g\right\rangle_{L^{2}}\right| \leq\|f\|_{\mathcal{H}}\left\|\mathcal{D}_{-}^{H-1 / 2} g\right\|
$$

The problem with 4.24 is that we would like to apply it to a function $f$ which is $\gamma$-Hölder continuous on $[0,1]$, but does not necessarily vanish at either 0 or 1 , so that $\mathcal{D}_{-}^{H-1 / 2} f$ does in general not belong to $L^{2}$. If we define however $h(t)=t^{\gamma}(1-t)^{\gamma}$ for $t \in[0,1]$ and $h(t)=0$ for $t \geq 1$, then $\|f h\|_{\gamma} \leq C\|f\|_{\gamma}$, but $f h$ vanishes at 0 and at 1 . In particular, this implies that $\left\|\mathcal{D}_{-}^{H-1 / 2} f h\right\| \leq C\|f\|_{\gamma}$, so that

$$
\|f\|_{\mathcal{H}} \geq C \frac{\int_{0}^{1} t^{\gamma}(1-t)^{\gamma} f^{2}(t) d t}{\|f\|_{\gamma}},
$$

for some constant $C$. On the other hand, it is a straightforward calculation to check that

$$
\int_{0}^{1} t^{\gamma}(1-t)^{\gamma} f^{2}(t) d t \geq C \frac{\|f\|_{\infty}^{3+1 / \gamma}}{\|f\|_{\gamma}^{1+1 / \gamma}},
$$

which implies the desired result.

Corollary 4.5 Let $y$ be a random process with sample paths that are almost surely $\gamma$-Hölder continuous for some $\gamma>H-1 / 2$. Then, there exists an exponent $\alpha$ such that

$$
\mathbb{P}\left(\|y\|_{\mathcal{H}}<\varepsilon\right) \leq \mathbb{P}\left(\|y\|_{L^{\infty}}<\varepsilon^{\alpha}\right)+\mathbb{P}\left(\|y\|_{\gamma}>\varepsilon^{-\alpha}\right),
$$

for every $\varepsilon$ sufficiently small. 
Proof. It follows from Lemma4.4 that

$$
\mathbb{P}\left(\|y\|_{\mathcal{H}}<\varepsilon\right) \leq \mathbb{P}\left(C\|y\|_{L^{\infty}}^{3+1 / \gamma}\|y\|_{\gamma}^{-2-1 / \gamma}<\varepsilon\right) .
$$

For an arbitrary pair of positive random variables $X$ and $Y$, one always has

$$
\mathbb{P}(X / Y<\varepsilon) \leq \mathbb{P}\left(X<\varepsilon^{1-\alpha}\right)+\mathbb{P}\left(Y>\varepsilon^{-\alpha}\right),
$$

so that the claim follows by taking $\alpha$ small enough.

This provides us with the necessary tools to complete the

Proof of Theorem 4.3 We shall show that $X_{1}^{x_{0}}$ admits a smooth density with respect to the Lebesgue measure, by using the Malliavin covariance matrix $\Gamma_{1}$ associated with $X_{1}^{x_{0}}$. Note that we can consider the case $t=1$ without any loss of generality by rescaling the vector fields $V_{i}$ appropriately.

Let $\Gamma_{1}$ be the Malliavin covariance matrix associated with $X_{1}^{x_{0}}$. By definition, we have

$$
\Gamma_{1}=\left(\left\langle\mathbf{D} X_{1}^{i, x_{0}}, \mathbf{D} X_{1}^{j, x_{0}}\right\rangle_{\mathcal{H}}\right)_{1 \leq i, j \leq n}
$$

To show that $X_{1}^{x_{0}}$ has a a smooth density, it suffices to show that with probability one $\Gamma_{1}$ is invertible and that for every $p>1$,

$$
\mathbb{E}\left(\frac{1}{\left|\operatorname{det} \Gamma_{1}\right|^{p}}\right)<+\infty
$$

From Lemma 4.2

$$
\mathbf{D}_{s}^{j} X_{1}^{x_{0}}=\mathbf{J}_{0 \rightarrow 1} \mathbf{J}_{0 \rightarrow s}^{-1} V_{j}\left(X_{s}\right), \quad j=1, \ldots, d, \quad 0 \leq s \leq 1 .
$$

Therefore,

$$
\Gamma_{1}=H(2 H-1) \mathbf{J}_{0 \rightarrow 1} \int_{0}^{1} \int_{0}^{1} \mathbf{J}_{0 \rightarrow u}^{-1} V\left(X_{u}^{x_{0}}\right) V\left(X_{v}^{x_{0}}\right)^{T}\left(\mathbf{J}_{0 \rightarrow v}^{-1}\right)^{T}|u-v|^{2 H-2} d u d v \mathbf{J}_{0 \rightarrow 1}^{T},
$$

where $V$ denotes the $n \times d$ matrix $\left(V_{1} \ldots V_{d}\right)$. Since $\mathbf{J}_{0 \rightarrow 1}$ is almost surely invertible with inverse in $L^{p}, p>1$, in order to show that $\Gamma_{1}$ is invertible with probability one, it is enough to check that with probability one, the matrix

$$
C_{1}=\int_{0}^{1} \int_{0}^{1} \mathbf{J}_{0 \rightarrow u}^{-1} V\left(X_{u}^{x_{0}}\right) V\left(X_{v}^{x_{0}}\right)^{T}\left(\mathbf{J}_{0 \rightarrow v}^{-1}\right)^{T}|u-v|^{2 H-2} d u d v
$$

is invertible and satisfies for every $p>1$,

$$
\mathbb{E}\left(\frac{1}{\left|\operatorname{det} C_{1}\right|^{p}}\right)<+\infty
$$


By using Proposition 3.4 the idea is now to control the smallest eigenvalue of $C_{1}$ by showing that it can not be too small. More precisely, recall (Lemma 2.3.1. in [Nua95]) that if for any $p \geq 2$, there exists $\epsilon_{0}(p)$ such that for every $\epsilon \leq \epsilon_{0}(p)$,

$$
\sup _{\|v\|=1} \mathbb{P}\left(\left\langle v, C_{1} v\right\rangle \leq \epsilon\right) \leq \epsilon^{p}
$$

then $C_{1}$ is invertible with probability one and 4.25) holds for every $p>1$. We thus want to estimate $\mathbb{P}\left(\left\langle v, C_{1} v\right\rangle \leq \epsilon\right)$. Let us observe that

$$
\begin{aligned}
\left\langle v, C_{1} v\right\rangle & =\sum_{j=1}^{d} \int_{0}^{1} \int_{0}^{1}|s-t|^{2 H-2}\left\langle v,\left(\Phi_{s}^{*} V_{j}\right)\left(x_{0}\right)\right\rangle\left\langle v,\left(\Phi_{t}^{*} V_{j}\right)\left(x_{0}\right)\right\rangle d s d t \\
& =\sum_{j=1}^{d}\left\|\left\langle v,\left(\Phi^{*} V_{i}\right)\left(x_{0}\right)\right\rangle\right\|_{\mathcal{H}}^{2} .
\end{aligned}
$$

Fix now an (arbitrarily large) value $p>0$. It follows from Lemma 4.1 and Corollary 4.5 that there exists $\alpha>0$ such that

$$
\mathbb{P}\left(\left\langle v, C_{1} v\right\rangle \leq \varepsilon\right) \leq C \varepsilon^{p}+\min _{i=1, \ldots, d} \mathbb{P}\left(\left\|\left\langle v,\left(\Phi^{*} V_{i}\right)\left(x_{0}\right)\right\rangle\right\|_{L^{\infty}} \leq \epsilon^{\alpha}\right) .
$$

Note now that if $V$ is an arbitrary bounded vector field with bounded derivatives, the chain rule reads

$$
\left(\Phi_{t}^{*} V\right)\left(x_{0}\right)=\int_{0}^{t}\left\langle y,\left(\Phi_{s}^{*}\left[V_{0}, V\right]\right)\left(x_{0}\right)\right\rangle d s+\sum_{j=1}^{d} \int_{0}^{t}\left\langle y,\left(\Phi_{s}^{*}\left[V_{j}, V\right]\right)\left(x_{0}\right)\right\rangle d B_{s}^{j} .
$$

It thus follows from Proposition 3.4 and Corollary 4.5 that there exists $\alpha$ such that

$$
\mathbb{P}\left(\left\|\left\langle v,\left(\Phi_{.}^{*} V\right)\left(x_{0}\right)\right\rangle\right\|_{L^{\infty}}<\varepsilon\right) \leq C \varepsilon^{p}+\min _{i=0, \ldots, d} \mathbb{P}\left(\left\|\left\langle v,\left(\Phi^{*}\left[V_{i}, V\right]\right)\left(x_{0}\right)\right\rangle\right\|_{L^{\infty}}<\varepsilon^{\alpha}\right)
$$

Consider now the integer $N$ from the assumption. Combining 4.26 with 4.27, we see that there exists $\alpha>0$ such that

$$
\mathbb{P}\left(\left\langle v, C_{1} v\right\rangle \leq \varepsilon\right) \leq C \varepsilon^{p}+\min _{V \in \overline{\mathcal{V}}_{N}} \mathbb{P}\left(\left\|\left\langle v,\left(\Phi^{*} V\right)\left(x_{0}\right)\right\rangle\right\|_{L^{\infty}} \leq \epsilon^{\alpha}\right) .
$$

for all $\epsilon$ small enough. On the other hand, we know by assumption that $\left\{V\left(x_{0}\right), V \in \overline{\mathcal{V}}_{N}\right\}$ spans all of $\mathbb{R}^{n}$, so that there exists some $V \in \overline{\mathcal{V}}_{N}$ such that $\left\langle v, V\left(x_{0}\right)\right\rangle \neq 0$. Therefore, one has $\mathbb{P}\left(\left\langle v, C_{1} v\right\rangle \leq \varepsilon\right) \leq C \varepsilon^{p}$ for all $\varepsilon$ sufficiently small, which is the required bound.

\section{Asymptotics of the density in small times}

In order to obtain asymptotics of the density of hypoelliptic diffusions on the diagonal in small times, one method consists to approximate the diffusion by the lift of the Brownian motion in a 
nilpotent Lie group that is called a Carnot group (see [Bau05] or [BA89]). In a recent work [BC06], Baudoin and Coutin have introduced and studied fractional Brownian motions on Carnot groups. We shall see that the solution of a stochastic differential equation driven by fractional Brownian motions can, under Hörmander's type assumptions, be approximated by fractional Brownian motions on Carnot groups. From this approximation, we will deduce an asymptotic development of the density in small times.

We recall first the notion of Carnot group (see e.g. [Bau05]) and the main results that are obtained in [BC06].

Definition 5.1 A Carnot group of step (or depth) $N$ is a simply connected Lie group $\mathbb{G}$ whose Lie algebra can be decomposed as

$$
\mathcal{V}_{1} \oplus \ldots \oplus \mathcal{V}_{N}
$$

where

$$
\left[\mathcal{V}_{i}, \mathcal{V}_{j}\right]=\mathcal{V}_{i+j}
$$

and

$$
\mathcal{V}_{s}=0, \text { for } s>N \text {. }
$$

Notice that the vector space $\mathcal{V}_{1}$, which is called the basis of $\mathbb{G}$, Lie generates $\mathfrak{g}$, where $\mathfrak{g}$ denotes the Lie algebra of $\mathbb{G}$. It is possible to show that for every $N \geq 1$, up to an isomorphism, there exists exactly one $N$-step nilpotent Carnot group with basis $\mathbb{R}^{d}$. This group shall be denoted by $\mathbb{G}_{N}\left(\mathbb{R}^{d}\right)$.

Since a Carnot group $\mathbb{G}$ is $N$-step nilpotent and simply connected, the exponential map is a diffeomorphism. On $\mathfrak{g}$ we can consider the family of linear operators $\delta_{t}: \mathfrak{g} \rightarrow \mathfrak{g}, t \geq 0$ which act by scalar multiplication $t^{i}$ on $\mathcal{V}_{i}$. These operators are Lie algebra automorphisms due to the grading. The maps $\delta_{t}$ induce Lie group automorphisms $\Delta_{t}: \mathbb{G} \rightarrow \mathbb{G}$ which are called the canonical dilations of $\mathbb{G}$. Let us now take a basis $U_{1}, \ldots, U_{d}$ of the vector space $\mathcal{V}_{1}$. The vectors $U_{i}$ can be seen as left invariant vector fields on $\mathbb{G}$ so that we can consider the following stochastic differential equation on $\mathbb{G}$ :

$$
d X_{t}=\sum_{i=1}^{d} \int_{0}^{t} U_{i}\left(X_{s}\right) d B_{s}^{i}, t \geq 0,
$$

which is easily seen to have a unique solution associated with the initial condition $X_{0}=1_{\mathbb{G}}$. The driving process $\left(B_{t}\right)_{t \geq 0}$ is here a fractional Brownian motion with Hurst parameter $H>1 / 2$. The process $\left(X_{t}\right)_{t \geq 0}$ is called the lift of $\left(B_{t}\right)_{t \geq 0}$ in the group $\mathbb{G}$. For this equation, the assumptions of Theorem 4.3 are obviously satisfied, so that we have a smooth density for $X_{t}, t>0$, with respect to the Haar measure of $\mathbb{G}$.

We have then the global scaling property

$$
\left(X_{c t}\right)_{t \geq 0} \stackrel{\text { law }}{=}\left(\Delta_{c^{H}} X_{t}\right)_{t \geq 0} .
$$

(See [BC06] for a proof.) This scaling property leads directly to the following value at $1_{\mathbb{G}}$ of the density $\tilde{p}_{t}$ of $X_{t}$ with respect to the Haar measure of $\mathbb{G}$ :

$$
\tilde{p}_{t}\left(1_{\mathbb{G}}\right)=\frac{C}{t^{D H}}, t>0,
$$


where $C>0$ and $D=\sum_{i=1}^{N} i \operatorname{dim} \mathcal{V}_{i}$. From this, we will see how to deduce asymptotics in small times for any hypoelliptic stochastic differential equation driven by fractional Brownian motions.

From now on, we consider $d$ vector fields $V_{i}: \mathbb{R}^{n} \rightarrow \mathbb{R}^{n}$ which are $C^{\infty}$ bounded and shall always assume that the following assumption is satisfied.

Strong Hörmander's Condition: For every $x \in \mathbb{R}^{n}$, we have:

$$
\operatorname{span}\left\{V_{I}(x), I \in \cup_{k \geq 1}\{1, \ldots, d\}^{k}\right\}=\mathbb{R}^{n} .
$$

We recall that if $I=\left(i_{1}, \ldots, i_{k}\right) \in\{1, \ldots, d\}^{k}$ is a word, we denote by $V_{I}$ the commutator defined by

$$
V_{I}=\left[V_{i_{1}},\left[V_{i_{2}}, \ldots,\left[V_{i_{k-1}}, V_{i_{k}}\right] \ldots\right] .\right.
$$

Let us introduce some concepts of differential geometry. The set of linear combinations with smooth coefficients of the vector fields $V_{1}, \ldots, V_{d}$ is called the differential system (or sheaf) generated by these vector fields. It shall be denoted by $\mathcal{D}$ in the sequel. Notice that $\mathcal{D}$ is naturally endowed with a $\mathcal{C}_{\infty}\left(\mathbb{R}^{n}, \mathbb{R}\right)$-module structure. For $x \in \mathbb{R}^{n}$, we denote

$$
\mathcal{D}(x)=\{X(x), X \in \mathcal{D}\} .
$$

If the integer $\operatorname{dim} \mathcal{D}(x)$ does not depend on $x$, then $\mathcal{D}$ is said to be a distribution. The Lie brackets of vector fields in $\mathcal{V}$ generate a flag of differential systems,

$$
\mathcal{D} \equiv \mathcal{D}^{1} \subset \mathcal{D}^{2} \subset \cdots \subset \mathcal{D}^{k} \subset \cdots,
$$

where $\mathcal{D}^{k}$ is recursively defined by the formula

$$
\mathcal{D}^{k}=\mathcal{D}^{k-1}+\left[\mathcal{D}, \mathcal{D}^{k-1}\right] .
$$

As a module, $\mathcal{D}^{k}$ is generated by the set of vector fields $V_{I}$, where $I$ describes the set of words with length $k$. Moreover, due to Jacobi identity, we have $\left[\mathcal{D}^{i}, \mathcal{D}^{j}\right] \subset \mathcal{D}^{i+j}$. This flag is called the canonical flag associated with the differential system $\mathcal{D}$. Hörmander's strong condition, which we supposed to hold, states that for each $x \in \mathbb{R}^{n}$, there is a smallest integer $r(x)$ such that $\mathcal{D}^{r(x)}=\mathbb{R}^{n}$. For each $x \in \mathbb{R}^{n}$, the canonical flag induces a flag of vector subspaces,

$$
\mathcal{D}(x) \subset \mathcal{D}^{2}(x) \subset \cdots \subset \mathcal{D}^{r(x)}(x)=\mathbb{R}^{n} .
$$

The integer list $\left(\operatorname{dim} \mathcal{D}^{k}(x)\right)_{1 \leq k \leq r(x)}$ is called the growth vector of $\mathcal{V}$ at $x$. The point $x$ is said to be a regular point of $\mathcal{V}$ if the growth vector is constant in a neighbourhood of $x$. Otherwise, we say that $x$ is a singular point. On a Carnot group, due to the homogeneity, all points are regular.

Let $\mathcal{V}_{i}=\mathcal{D}^{i} / \mathcal{D}^{i-1}$ denote the quotient differential systems, and define

$$
\mathcal{N}(\mathcal{D})=\mathcal{V}_{1} \oplus \cdots \oplus \mathcal{V}_{k} \oplus \cdots
$$

The Lie bracket of vector fields induces a bilinear map on $\mathcal{N}(\mathcal{D})$ which respects the grading: $\left[\mathcal{V}_{i}, \mathcal{V}_{j}\right] \subset \mathcal{V}_{i+j}$. Actually, $\mathcal{N}(\mathcal{D})$ inherits the structure of a sheaf of Lie algebras. Moreover, if $x$ is a regular point of $\mathcal{D}$, then this bracket induces a $r(x)$-step nilpotent graded Lie algebra structure on $\mathcal{N}(\mathcal{D})(x)$. Observe that the dimension of $\mathcal{N}(\mathcal{D})(x)$ is equal to $n$ and that from the definition, $\left(V_{1}(x), \ldots, V_{d}(x)\right)$ Lie generates $\mathcal{N}(\mathcal{D})(x)$. 
Definition 5.2 If $x$ is a regular point of $\mathcal{D}$, the $r(x)$-step nilpotent graded Lie algebra $\mathcal{N}(\mathcal{D})(x)$ is called the nilpotentisation of $\mathcal{D}$ at $x$. This Lie algebra is the Lie algebra of a unique Carnot group which shall be denoted $\operatorname{Gr}(\mathcal{D})(x)$ and called the tangent space to $\mathcal{D}$ at $x$. The integer $D=\sum_{k=1}^{r(x)} k \operatorname{dim} \mathcal{D}^{k}(x)$ is called the homogeneous dimension of $\operatorname{Gr}(\mathcal{D})(x)$.

Proposition 5.3 Let $x$ be a regular point of $\mathcal{D}$. Let $p_{t}, t>0$, denote the density with respect to the Lebesgue measure of the solution of the stochastic differential equation

$$
X_{t}^{x}=x+\sum_{i=1}^{d} \int_{0}^{t} V_{i}\left(X_{s}^{x}\right) d B_{s}^{i}
$$

where $\left(B_{t}\right)_{t \geq 0}$ is a d-dimensional fractional Brownian motion with Hurst parameter $H>1 / 2$. We have,

$$
p_{t}(x) \sim_{t \rightarrow 0} \frac{C(x)}{t^{H D(x)}},
$$

where $C(x)$ is a non negative constant and $D(x)$ the homogeneous dimension of the tangent space $\operatorname{Gr}(\mathcal{D})(x)$.

Proof. Let us first introduce some notations: For $k \geq 1$, we denote by $\Delta^{k}[0, t]$ the simplex of ordered $k$-tuples with values in $[0, t]$, i.e.

$$
\Delta^{k}[0, t]=\left\{\left(t_{1}, \ldots, t_{k}\right) \in[0, t]^{k}, t_{1}<\ldots<t_{k}\right\} ;
$$

If $I=\left(i_{1}, \ldots, i_{k}\right) \in\{1, \ldots, d\}^{k}$ is a word with length $k$, we define the corresponding iterated integral of $B$ by

$$
\int_{\Delta^{k}[0, t]} d B^{I}=\int_{0<t_{1}<\ldots<t_{k}<t} d B_{t_{1}}^{i_{1}} \cdots d B_{t_{k}}^{i_{k}}
$$

where the right hand side consists of nested Riemann-Stieltjes integrals.

We denote $\mathfrak{S}_{k}$ the group of the permutations of the index set $\{1, \ldots, k\}$ and if $\sigma \in \mathfrak{S}_{k}$ and $I$ is a word $I=\left(i_{1}, \ldots, i_{k}\right)$, we denote by $\sigma \cdot I$ the word $\left(i_{\sigma(1)}, \ldots, i_{\sigma(k)}\right)$.

If $\sigma \in \mathfrak{S}_{k}$, we denote $e(\sigma)$ the cardinality of the set

$$
\{j \in\{1, \ldots, k-1\}, \sigma(j)>\sigma(j+1)\},
$$

i.e. $e(\sigma)$ is the number of raising sequences of $\sigma$. Finally, if $I=\left(i_{1}, \ldots, i_{k}\right) \in\{1, \ldots, d\}^{k}$ is a word

$$
\Lambda_{I}(B)_{t}=\sum_{\sigma \in \mathfrak{S}_{k}} \frac{(-1)^{e(\sigma)}}{k^{2}\left(\begin{array}{c}
k-1 \\
e(\sigma)
\end{array}\right)} \int_{\Delta^{k}[0, t]} d B^{\sigma^{-1} \cdot I}
$$

As a consequence of Proposition 23 in [FV06], we get the following approximation result

$$
X_{t}^{x}=\left[\exp \left(\sum_{k=1}^{r(x)} \sum_{I=\left(i_{1}, \ldots, i_{k}\right)} \Lambda_{I}(B)_{t} V_{I}\right)\right](x)+t^{\frac{r(x)+1}{2}} \mathbf{R}(t), t \geq 0 .
$$


Here, the remainder term $\mathbf{R}(t)$ is such that there exist $\alpha, c>0$ such that, for all $A>c$,

$$
\limsup _{t \rightarrow 0} \mathbb{P}\left(\sup _{0 \leq s \leq t} s^{H(N+1)}\left|\mathbf{R}_{N}(s)\right| \geq A t^{H(N+1)}\right) \leq \exp \left(-\frac{A^{\alpha}}{c}\right) .
$$

It is shown in [Bau05, Proposition 3.7, pp 66] that one can write

$$
\left[\exp \left(\sum_{k=1}^{r(x)} \sum_{I=\left(i_{1}, \ldots, i_{k}\right)} \Lambda_{I}(B)_{t} V_{I}\right)\right](x)=F\left(B_{t}^{*}\right)
$$

where $\left(B_{t}^{*}\right)_{t \geq 0}$ is the lift of $\left(B_{t}\right)_{t \geq 0}$ in the free Carnot group $\mathbb{G}_{r(x)}\left(\mathbb{R}^{d}\right)$ and where $F$ is a map

$$
\mathbb{G}_{r(x)}\left(\mathbb{R}^{d}\right) \rightarrow \mathbb{R}^{n}
$$

that is Pansu differentiable at $1_{\mathbb{G}_{r(x)}}$. Furthermore, its Pansu's derivative

$$
d_{P} F\left(1_{\mathbb{G}_{r(x)}}\right): \mathbb{G}_{r(x)}\left(\mathbb{R}^{d}\right) \rightarrow \operatorname{Gr}(\mathcal{D})(x)
$$

is a surjective Carnot group morphism. Now, we have

$$
\left(F\left(B_{t}^{*}\right)\right)_{t \geq 0} \stackrel{\text { law }}{=}\left(F\left(\Delta_{\sqrt{t}}^{\mathbb{G}_{r(x)}\left(\mathbb{R}^{d}\right)} B_{1}^{*}\right)\right)_{t \geq 0},
$$

and

$$
F\left(\Delta_{\sqrt{t}}^{\mathbb{G}_{r(x)}\left(\mathbb{R}^{d}\right)} B_{1}^{*}\right) \approx x \Delta_{\sqrt{t}}^{\operatorname{Gr}(\mathcal{D})(x)} d_{P} F\left(0_{\mathbb{G}_{r(x)}\left(\mathbb{R}^{d}\right)}\right)\left(B_{1}^{*}\right)
$$

in small times. Since $d_{P} F\left(0_{\mathbb{G}_{r(x)}}\left(\mathbb{R}^{d}\right)\right)$ is surjective, the random variable

$$
d_{P} F\left(0_{\mathbb{G}_{r(x)}\left(\mathbb{R}^{d}\right)}\right)\left(B_{1}^{*}\right)
$$

admits a density with respect to the Lebesgue measure on $\mathbb{R}^{n}$. This concludes the proof.

\section{References}

[BA89] G. BEN Arous. Développement asymptotique du noyau de la chaleur hypoelliptique sur la diagonale. Annales de l'institut Fourier 39, (1989), 73-99.

[Bau05] F. BAudoin. An Introduction to the Geometry of Stochastic Flows. Imperial College Press, 2005.

[BC06] F. BAUdoin and L. Coutin. Self-similarity and fractional brownian motions on lie groups, 2006. Preprint. 
[Bis81] J.-M. BismUT. Martingales, the Malliavin calculus and hypoellipticity under general Hörmander's conditions. Z. Wahrsch. Verw. Gebiete 56, no. 4, (1981), 469-505.

[Bog98] V. I. Bogachev. Gaussian measures, vol. 62 of Mathematical Surveys and Monographs. American Mathematical Society, Providence, RI, 1998.

[CIS76] B. S. Cirel'son, I. A. Ibragimov, and V. N. Sudakov. Norms of Gaussian sample functions. In Proceedings of the Third Japan-USSR Symposium on Probability Theory (Tashkent, 1975), 20-41. Lecture Notes in Math., Vol. 550. Springer, Berlin, 1976.

[CQ02] L. Coutin and Z. QIAN. Stochastic analysis, rough path analysis and fractional Brownian motions. Probab. Theory Related Fields 122, no. 1, (2002), 108-140.

[FV06] P. Friz and N. Victoir. Euler estimates for rough differential equations, 2006. Preprint.

[HN06] Y. Hu and D. NuAlaRT. Differential equations driven by hölder continuous functions of order greater than 1/2, 2006. Preprint.

[Hör67] L. Hörmander. Hypoelliptic second order differential equations. Acta Math. 119, (1967), 147-171.

[Mal78] P. Malliavin. Stochastic calculus of variations and hypoelliptic operators. Symp. on Stoch. Diff. Equations, Kyoto 1976 147-171.

[Nor86] J. NorRIS. Simplified Malliavin calculus. In Séminaire de Probabilités, XX, 1984/85, vol. 1204 of Lecture Notes in Math., 101-130. Springer, Berlin, 1986.

[NR02] D. NUAlaRT and A. RĂŞCANU. Differential equations driven by fractional Brownian motion. Collect. Math. 53, no. 1, (2002), 55-81.

[NS05] D. NuAlarT and B. SAUSSEREAU. Malliavin calculus for stochastic differential equations driven by a fractional brownian motion, 2005. Preprint.

[NT06] I. NOURDIN and S. T. On the absolute continuity of one-dimensional sdes driven by a fractional brownian motion. Statistics and Probability Letters 76, (2006), 907-912.

[Nua95] D. Nualart. The Malliavin calculus and related topics. Probability and its Applications (New York). Springer-Verlag, New York, 1995.

[PT00] V. PIPIRAS and M. S. TAQQU. Integration questions related to fractional Brownian motion. Probab. Theory Related Fields 118, no. 2, (2000), 251-291.

[SKM93] S. G. SAmKo, A. A. Kilbas, and O. I. MARICheV. Fractional integrals and derivatives. Gordon and Breach Science Publishers, Yverdon, 1993. Theory and applications, Edited and with a foreword by S. M. Nikol'skiǔ, Translated from the 1987 Russian original, Revised by the authors. 
[Ta195] M. TALAGRAND. Concentration of measure and isoperimetric inequalities in product spaces. Inst. Hautes Études Sci. Publ. Math. 81, (1995), 73-205.

[You36] L. C. Young. An inequality of Hölder type connected with Stieltjes integration. Acta Math. 67, (1936), 251-282.

[Zäh01] M. ZäHLE. Integration with respect to fractal functions and stochastic calculus. II. Mathematische Nachrichten 225, (2001), 145-183. 